\title{
Diagnostic Value of Nineteen Different Imaging Methods for Patients with Breast Cancer: a Network Meta-Analysis
}

\author{
Xiao-Hong Zhang Can Xiao \\ Department of Ultrasound, Huaihe Hospital of Henan University, Kaifeng, P.R. China
}

\section{Key Words}

Breast cancer - Diagnostic value - Magnetic resonance imaging - Mammography . Ultrasonography $\bullet$ Scintimammography $\bullet$ Sensitivity $・$ Network meta-analysis

\begin{abstract}
Background/Aims: We performed a network meta-analysis (NMA) to investigate and compare the diagnostic value of 19 different imaging methods used for breast cancer (BC). Methods: Cochrane Library, PubMed and EMBASE were searched to collect the relevant literature from the inception of the study until November 2016. A combination of direct and indirect comparisons was performed using an NMA to evaluate the combined odd ratios (OR) and draw the surface under the cumulative ranking curves (SUCRA) of the diagnostic value of different imaging methods for BC. Results: A total of 39 eligible diagnostic tests regarding 19 imaging methods (mammography [MG], breast-specific gamma imaging [BSGI], color Doppler sonography $[C D]$, contrast-enhanced magnetic resonance imaging [CE-MRI], digital breast tomosynthesis [DBT], fluorodeoxyglucose positron-emission tomography/computed tomography [FDG PET/CT], fluorodeoxyglucose positron-emission tomography [FDG-PET], full field digital mammography [FFDM], handheld breast ultrasound [HHUS], magnetic resonance imaging [MRI], automated breast volume scanner [ABUS], magnetic resonance mammography $[\mathrm{MRM}]$, scintimammography $[\mathrm{SMM}]$, single photon emission computed tomography scintimammography [SPECT SMM], ultrasound elastography [UE], ultrasonography [US], mammography + ultrasonography [MG + US], mammography + scintimammography [MG + SMM], and ultrasound elastography + ultrasonography [UE + US]) were included in the study. According to this network meta-analysis, in comparison to the MG method, the CE-MRI, MRI, MRM, MG + SMM and UE + US methods exhibited relatively higher sensitivity, and the specificity of the FDG PET/CT method was higher, while the BSGI and MRI methods exhibited higher accuracy. Conclusion: The results from this NMA indicate that the diagnostic value of the BSGI, MG + SMM, MRI and CE-MRI methods for BC were relatively higher in terms of sensitivity, specificity and accuracy.
\end{abstract}

X.-H. Zhang and C. Xiao contributed equally to this work. 


\section{Introduction}

Breast cancer (BC) is the most commonly diagnosed cancer in women, and approximately 1.7 million new cases of $\mathrm{BC}$ and 0.5 million cancer deaths owing to $\mathrm{BC}$ were recorded worldwide in 2012 [1]. BC ranks first among the cancers diagnosed in women between 20 and 59 years of age [2]. During the past 30 years, BC mortality in Chinese females has followed a gradual upward trend, making it the fifth most common cause of cancer death in females [1]. BC is also a huge financial burden and source of pain in patients' daily lives [3]. The clinical outcome of $\mathrm{BC}$ is immensely variable, ranging from complete curability to a time span of 10 years post-surgical treatment, owing to the heterogeneous group of tumors presented with $\mathrm{BC}[4,5]$. The complicated scenarios arising in $\mathrm{BC}$ can be explained by its complex neoplasm and both genetic and environmental factors [6]. In addition, family history, high breast density, overweight, and environmental factors, such as X- and $\gamma$-radiation, hormones and breast-feeding, play a key part in contributing to this complicated scenario [7]. The ultimate outcome of BC relies on its initial stage at diagnosis, with the main prognostic factors being lymph node involvement, tumor size and histological grade [8]. However, the heterogeneous tumors of $\mathrm{BC}$ can behave in different manners even at the same stage, and the prognosis varies from individual to individual [9]. BC has been classified into the following five molecular subtypes: luminal A, luminal B, human epidermal growth factor receptor-2 (HER-2) overexpressing, basal-like, and normal-like [10]. Complex tumor therapy development and new opportunities in surgery, which take into account both oncological principles and esthetic aspects, have set the requirements far higher for diagnostic imaging of the breast and for radiologists [11].

Great strides have been achieved in screening for BC over the past decades. The improved awareness and screening have brought about early diagnosis in BC patients, which in turn contributes to higher survival rates in female BC patients [12]. Diagnostic methods for BC are classified as magnetic resonance imaging (MRI), mammography (MG), ultrasonography (US), scintimammography (SMM), and bioluminescence imaging (BLI) $[13,14]$.. In both in situ and invasive BC, the increasingly used method of diagnosis is the MRI [15]. Nevertheless, the use of breast MRI remains controversial because of the debate regarding the long-term clinical benefits from surgical excision of additional tumor detected by MRI in the ipsilateral breast $[16,17]$. Additionally, the randomized trial of preoperative MRI does not effectively affect the frequency of avoidable operations (total initial mastectomies not justified by pathology, and re-excisions and mastectomies within six months after breast-conserving surgery] [18, 19]. Therefore, we require additional imaging examinations to detect the exact location and extension of recurrent lesions [20]. Other imaging methods, such as MG, US, and SMM, as well as combined diagnosis, also present their own set of advantages and disadvantages in sensitivity, specificity and accuracy in the diagnosis of BC [11]. The statistical factors in reviews that compare various interventions and network meta-analysis (NMA), if properly used, can exert decision-making better than the pairwise meta-analysis [21].

The exact pre-therapeutic tumor sizing using imaging methods plays a central role [13], and NMA is a relatively new statistical technique that gives access to compare both direct and indirect evidence [22]. NMA shows further development in evidence synthesis, particularly in the decision-making field, and it not only combines data from random comparisons to offer an internally consistent estimation but also realizes randomization in the evidence [23, 24]. Therefore, we conducted an NMA to analyze the sensitivity, specificity and accuracy of different imaging methods to find the most accurate and efficient method in the diagnosis of BC.

\section{Materials and Methods}

Search strategy

The full text is written in accordance with a network meta-analysis of the PRISMA declaration [25]. We accessed the Cochrane Library, PubMed and EMBASE databases to obtain literature relevant to this 
study. Relevant articles were also reviewed manually in case of the omission of any potentially relevant literature. The literature search was limited to the English language and ended in November 2016. The search terms included a combination of keywords and free words as follows: breast cancer (BC), magnetic resonance imaging (MRI), mammography (MG), computed tomography (CT), ultrasonography (US), scintimammography (SMM), ultrasound elastography (UE), digital breast tomosynthesis (DBT), color Doppler sonography (CD), breast-specific gamma imaging (BSGI), automated breast volume scanner (ABUS), sensitivity, comparative study, sonography, mammography, elastography and diagnosis. The detailed search strategies are shown (for all online suppl. material, see www.karger.com/10.1159/000489443) in Suppl. material.

\section{Inclusion and exclusion criteria}

The inclusion criteria for the study were as follows: (1) the study designs must be diagnostic tests; (2) the imaging methods were limited to MG (used with a mammography system), BSGI (conducted with a highresolution breast-specific gamma camera), CD, contrast-enhanced magnetic resonance imaging (CE-MRI, using a $3 \mathrm{~T}$ MRI scanner in the prone position applying a dedicated four-channel breast coil), DBT (performed with a commercially available device comprising of a custom-designed high-power (mA) tungsten (W) anode X-ray tube and rhodium, silver, and aluminum X-ray filters), fluorodeoxyglucose positron-emission tomography/computed tomography (FDG PET/CT, using a combined whole-body PET/CT in-line system with a high-resolution PET scanner and a 64-row detector CT scanner), fluorodeoxyglucose positronemission tomography (FDG-PET, performed with 3D acquisition on a special partial-ring PET scanner in the prone position on a special device allowing the breast to depend freely), full field digital mammography (FFDM, conducted with a tomosynthesis capable unit), handheld breast ultrasound (HHUS, using a 13.5 MHz transducer), MRI, ABUS (with scanning unit, including a $14 \mathrm{MHz}$ high-frequency linear transducer), magnetic resonance mammography (MRM, regarded as Breast MRI), SMM (with a single-detector gamma camera including a parallel-hole low-energy high-resolution collimator), single photon emission computed tomography scintimammography (SPECT SMM), UE (complementary to and independent from B-mode and Doppler imaging, which offer the acoustic impedance and vascular flow information), US, mammography + ultrasonography (MG + US, mammography in comparison to ultrasonography), mammography + scintimammography ( $M G+S M M$, mammography in comparison to scintimammography), and ultrasound elastography + ultrasonography (UE + US, ultrasound elastography in comparison to ultrasonography); (3) study subjects were consecutive patients aging from 12 to 92 years with a possible breast lesion detected by self-examination, physical examination or screening mammography, and patients underwent examination before final diagnosis and confirmation by histopathologic examination; and (4) the outcome indicators of studies include sensitivity, specificity, positive predictive value (PPV), negative predictive value (NPV) and accuracy. The exclusion criteria included were as follows: (1) literature lacking data integrity (such as non-pairwise studies); (2) duplicate publications; (3) conference reports, systematic reviews and summary articles; (4) studies unrelated to BC; (5) non-English studies; and (6) non-human studies.

\section{Data extraction and quality assessment}

Two researchers independently carried out data extracted on the basis of predefined form. The main data included the primary author, date of publication, country, ethnicity, age, gender, gold standard and diagnostic modalities. Any dispute appearing during data extraction was resolved through discussion with 2 researchers. The quality of all included studies was assessed by researchers, according to the Quality Assessment of Diagnostic Accuracy Studies-2 (QUADAS-2) tool [26]. The QUADAS-2 tool included the following 4 key domains: patient selection, index test, reference standard, and flow of patients through the study and timing of the index tests and reference standard (flow and timing). The Review Manager 5 (RevMan 5.2.3, Cochrane Collaboration, Oxford, UK) statistical computing software was used to carry out quality assessment and investigation of publication bias.

\section{Statistical analysis}

Firstly, traditional pairwise meta-analyses were performed for studies to compare different diagnostic modalities using the Stata version 13.1 software (StataCorp, College Station, TX, USA). The pooled estimates of odd ratios (ORs) and 95\% confidence intervals (CI) for sensitivity, specificity, PPV, NPV and accuracy of BC were shown. Heterogeneity among studies was tested using the I-square and Chi-square tests [27]. Secondly, 
the $\mathrm{R}$ version 3.2.1 statistical computing software and network package were used to draw the network graphs, with each node representing different interventions, the node size reflecting the number of patients, and the thickness of lines between nodes indicating the number of included studies. Thirdly, Bayesian network meta-analyses were performed to combine the effective sizes of direct and indirect comparisons. Each analysis was based on non-informative priors for precision and effective sizes. Lack of auto-correlation and convergence were checked and confirmed by four chains and a 20, 000-simulation burn-in phase; finally, direct probability statements were derived from an additional 50, 000-simulation phase [28]. The node-splitting method was adopted to evaluate the consistency between direct and indirect evidence, and the consistency or inconsistency model was selected based on the results of the aforementioned evaluation [29]. To provide assistance in the interpretation of ORs, the surface under the cumulative ranking curve (SUCRA) was used to calculate the probability of each intervention, being the most effective diagnostic method based on a Bayesian approach using probability values, and the larger the SUCRA value is, the better the rank of the intervention [30,31]. Cluster analyses were conducted to evaluate different imaging methods on the diagnostic value of $\mathrm{BC}$ treatment, by grouping different interventions according to similarities of two variables to judge the efficacies by comparing the advantages and disadvantages of different imaging methods [30]. Comparison-adjusted funnel plots were performed to detect the small study effects on data $[30,32]$. R (V.3.2.1) package gemtc (V.0.6), along with the Markov Chain Monte Carlo engine Open BUGS (V.3.4.0), was used for all computations in this network meta-analysis.

\section{Results}

\section{Baseline characteristics of included studies}

A total of 5, 446 studies relevant to this study were initially retrieved. After excluding duplicate studies $(n=46)$, letters or reviews $(n=159)$, non-human studies $(n=156)$ and non-English studies $(\mathrm{n}=466)$, a total of 4, 719 studies were evaluated for eligibility by fulltext review. After full-text review, case-control studies $(n=2717)$, studies unrelated to BC ( $\mathrm{n}$ $=1318)$, studies irrelevant to imaging methods $(n=642)$, and studies lacking data or with incomplete data $(n=3)$ were ruled out. Finally, we identified 39 diagnostic tests that met the inclusion criteria [33-71] (Fig. 1), including a total of 2, 890 patients diagnosed with BC using the gold standard and 19 imaging methods, among which MG method was the most widely adopted method of diagnosis. The included studies were published between 1996 and 2015. In addition, 25 of the 39 enrolled studies were two-arm trials, 8 studies were three-arm trials, and 6 studies were fourarm trials. The baseline characteristics of the included studies are shown in Table 1, and the QUADAS-2 for evaluating the risk of bias is shown in Fig. 2. The results indicated that variations in test technology, execution, or interpretation may exert influence on the estimation of the diagnostic accuracy of a test.

Pairwise meta-analysis for the diagnostic value of 19 imaging methods for $B C$

We conducted a direct-paired comparison of the diagnostic value of 19 different imaging methods for the treatment of $\mathrm{BC}$. The results revealed

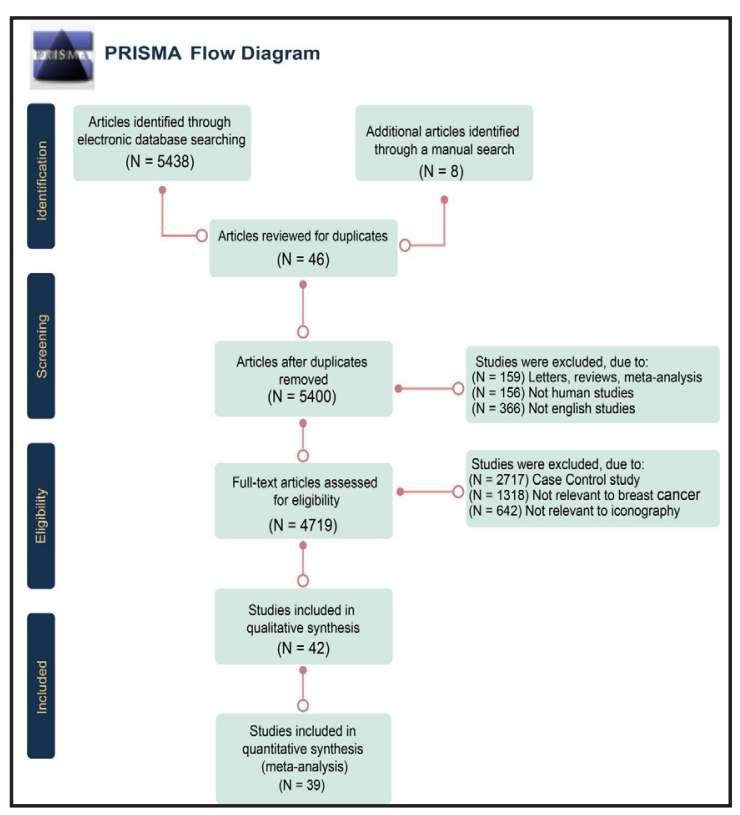

Fig. 1. Flow chart showing literature search and study selection with 39 relevant studies ultimately enrolled in our network meta-analysis. 
Table 1. Baseline characteristics of included studies. Note: $M=$ method; $N R=$ not reported; $A=$ mammography; $\mathrm{B}=$ breast-specific gamma imaging; $\mathrm{C}=$ Color Doppler sonography; $\mathrm{D}=$ contrast-enhanced magnetic resonance imaging; $\mathrm{E}$ = digital breast tomosynthesis; $\mathrm{F}$ = fluorodeoxyglucose positron-emission tomography/computed tomography; $\mathrm{G}$ = fluorodeoxyglucose positron-emission tomography; $\mathrm{H}$ = full field digital mammography; I = handheld breast ultrasound; $\mathrm{J}=$ magnetic resonance imaging; $\mathrm{K}=$ automated breast volume scanner; $\mathrm{L}=$ magnetic resonance mammography; $\mathrm{M}$ = scintimammography; $\mathrm{N}$ = single photon emission computed tomography scintimammography; $\mathrm{O}=$ ultrasound elastography; $\mathrm{P}=$ ultrasonography; $\mathrm{Q}=$ mammography+ultrasonography; $\mathrm{R}=$ mammography + scintimammography; $\mathrm{S}=$ ultrasound elastography + ultrasonography; a and b are different articles written by Kim BS in 2012

\begin{tabular}{|c|c|c|c|c|c|c|c|c|c|}
\hline \multirow{2}{*}{ Author } & \multirow{2}{*}{ Year } & \multirow{2}{*}{ Country } & \multirow{2}{*}{ Standard method } & \multirow{2}{*}{ Patient numbers } & \multirow{2}{*}{ Age } & \multicolumn{4}{|c|}{ Diagnostic modalities } \\
\hline & & & & & & M1 & M2 & M3 & M4 \\
\hline Palmedo H [71] & 1996 & Germany & biopsy & 56 & $58(22-81)$ & $\mathrm{M}$ & $\mathrm{A}$ & $\mathrm{J}$ & - \\
\hline Bone B [70] & 1997 & Sweden & biopsy & 238 & $52(23-83)$ & A & $\mathrm{J}$ & - & - \\
\hline Helbich TH [69] & 1997 & Austria & biopsy & 75 & $47(19-85)$ & $\mathrm{J}$ & $\mathrm{N}$ & - & - \\
\hline Blohmer JU [68] & 1999 & Germany & biopsy & 168 & NR & $\mathrm{C}$ & A & $\mathrm{P}$ & $\mathrm{J}$ \\
\hline Alamo L [67] & 2001 & Germany & cytology & 40 & $47(32-52)$ & $\mathrm{C}$ & $\mathrm{D}$ & - & - \\
\hline Lumachi F [66] & 2001 & Italy & biopsy & 87 & $48(26-79)$ & A & M & $\mathrm{R}$ & - \\
\hline Malich A [65] & 2001 & Germany & biopsy & 100 & NR & $\mathrm{P}$ & $\mathrm{L}$ & - & - \\
\hline Kolb TM [64] & 2002 & USA & biopsy & 27825 & $59.6(44-75)$ & $\mathrm{A}$ & $\mathrm{P}$ & - & - \\
\hline Bagni B [63] & 2003 & Italy & biopsy & 45 & $51(32-85)$ & M & $\mathrm{J}$ & - & - \\
\hline Bone B [62] & 2003 & Sweden & histopathology & 111 & $54(33-81)$ & $\mathrm{A}$ & M & $\mathrm{D}$ & M \\
\hline Chen DR [61] & 2003 & China & biopsy & 32 & $44.6(29-62)$ & $\mathrm{N}$ & $\mathrm{P}$ & - & - \\
\hline Goerres GW [60] & $2003 S$ & Switzerland & Cytology or histology & 40 & $57.2(32-76)$ & $G$ & $\mathrm{~J}$ & - & - \\
\hline Heinisch M [59] & 2003 & Australia & histopathology & 473 & $48.4(25-77)$ & $G$ & $\mathrm{D}$ & - & - \\
\hline Houssami N [58] & 2003 & Austria & biopsy & 258 & $40(22-55)$ & $\mathrm{P}$ & A & - & - \\
\hline Berg WA [57] & 2004 & USA & biopsy & 33 & $48.7(26-81)$ & $\mathrm{A}$ & $\mathrm{P}$ & $\mathrm{Q}$ & $\mathrm{J}$ \\
\hline Brem RF [56] & 2007 & USA & biopsy & 296 & $53(33-70)$ & B & $\mathrm{J}$ & - & - \\
\hline Zhi H [55] & 2007 & China & biopsy or fine-needle aspiration cytology & 97 & $42(17-87)$ & A & $\mathrm{P}$ & 0 & $\mathrm{~S}$ \\
\hline Pediconi F [54] & 2009 & Italy & histopathology & 78 & $47.5(16-77)$ & $\mathrm{J}$ & A & $\mathrm{P}$ & - \\
\hline Vassiou K [53] & 2009 & Greece & biopsy & 251 & $53(39-68)$ & $\mathrm{A}$ & $\mathrm{P}$ & $\mathrm{L}$ & $\mathrm{Q}$ \\
\hline Benndorf M [52] & 2010 & Germany & biopsy & 97 & $54.4(42-66)$ & $\mathrm{A}$ & $\mathrm{J}$ & - & - \\
\hline Hatzung G [51] & 2010 & Germany & biopsy & 110 & $54.8(21-92)$ & $\mathrm{P}$ & $A$ & $\mathrm{O}$ & $\mathrm{S}$ \\
\hline Leong LC [50] & 2010 & Singapore & biopsy & 90 & $46.7(18-74)$ & $\mathrm{P}$ & $\mathrm{O}$ & $\mathrm{S}$ & - \\
\hline Moy L [49] & 2010 & USA & biopsy & 242 & $43(24-65)$ & $\mathrm{J}$ & $\mathrm{F}$ & - & - \\
\hline Liew PL [48] & 2011 & China & fine-needle aspiration cytology & 351 & $46.2(12-87)$ & $\mathrm{A}$ & $\mathrm{P}$ & - & - \\
\hline Spangler ML [47] & 2011 & USA & biopsy & 299 & NR & $\mathrm{H}$ & $\mathrm{E}$ & - & - \\
\hline Svane G [46] & 2011 & Sweden & histopathology & 144 & $56.8(40-85)$ & A & $\mathrm{E}$ & - & - \\
\hline Kim BS-a [45] & 2012 & Korea & biopsy & 97.059 & $44.1(36-52)$ & $\mathrm{B}$ & $\mathrm{J}$ & - & - \\
\hline Kim BS-b [44] & 2012 & Korea & biopsy & 228 & $45.0(37-53)$ & $\mathrm{B}$ & $A$ & $\mathrm{P}$ & - \\
\hline Michell MJ [43] & 2012 & UK & biopsy & 759 & NR & $\mathrm{H}$ & $\mathrm{E}$ & - & - \\
\hline Svahn TM [42] & 2012 & USA & biopsy & 191 & $60(42-79)$ & $\mathrm{E}$ & A & - & - \\
\hline Golatta M [41] & 2013 & Germany & biopsy & 51 & $51(33-83)$ & $\mathrm{K}$ & I & A & - \\
\hline Shao H [40] & 2013 & China & pathological report & 90 & $53.2(26-85)$ & $\mathrm{A}$ & $\mathrm{J}$ & $\mathrm{P}$ & - \\
\hline Thibault F [39] & 2013 & France & biopsy & 131 & $61(40-90)$ & E & $\mathrm{H}$ & - & - \\
\hline Choi WJ [38] & 2014 & Korea & biopsy & 3700 & $47(19-82)$ & $\mathrm{K}$ & I & - & - \\
\hline Grigoryev M [37] & 2014 & Germany & biopsy & 105 & 51 & $\mathrm{P}$ & A & - & - \\
\hline Chou CP [36] & 2015 & China & biopsy & 59 & $53.3(40-64)$ & $\mathrm{J}$ & $\mathrm{F}$ & $\mathrm{P}$ & - \\
\hline Kim SA [35] & 2015 & Korea & biopsy & 118.643 & $49,6(29-78)$ & $E$ & $\mathrm{P}$ & - & - \\
\hline Magometschnigg HF [34] & 2015 & Germany & biopsy & 172 & $57(18-87)$ & $\mathrm{F}$ & $\mathrm{D}$ & - & - \\
\hline Moon $\mathrm{HJ}[33]$ & 2015 & Korea & biopsy & 4394 & $53.8(40-87)$ & $\mathrm{A}$ & $\mathrm{P}$ & - & - \\
\hline
\end{tabular}

that compared with the MG method, the BSGI method exhibited higher sensitivity and NPV, as well as lower accuracy, whereas the MRI method exhibited higher sensitivity, NPV and accuracy; the sensitivity and NPV of the UE + US and MRM methods were relatively higher; the SMM method presented relatively higher specificity, PPV and accuracy; and the US method exhibited lower specificity, PPV and accuracy and higher sensitivity. The results also demonstrated that the MG + US method exhibited higher specificity and PPV and lower sensitivity and NPV, whereas the MG + SMM method exhibited higher sensitivity and NPV (Table 2).

\section{Evidence network of 19 imaging methods for $B C$}

This study includes 19 imaging methods: $\mathrm{A}=$ mammography; $\mathrm{B}=$ breast-specific gamma imaging; $\mathrm{C}=$ color Doppler sonography; $\mathrm{D}=$ contrast-enhanced magnetic resonance imaging; $\mathrm{E}=$ digital breast tomosynthesis; $\mathrm{F}$ = fluorodeoxyglucose positron-emission tomography/ computed tomography; $\mathrm{G}=$ fluorodeoxyglucose positron-emission tomography; $\mathrm{H}=$ full field digital mammography; $\mathrm{I}=$ handheld breast ultrasound; $\mathrm{J}$ = magnetic resonance 
Table 2. Estimated OR and $95 \%$ CI of pairwise metaanalysis for sensitivity, specificity, PPV, NPV and accuracy for breast cancer. Note: $\mathrm{NA}=$ not available; $\mathrm{A}=$ mammography; $\mathrm{B}=$ breast-specific gamma imaging; $\mathrm{C}$ = Color Doppler sonography; $\mathrm{D}=$ contrast-enhanced magnetic resonance imaging; $\mathrm{E}=$ digital breast tomosynthesis; $\mathrm{F}$ = fluorodeoxyglucose positron-emission tomography/computed tomography; $\mathrm{G}$ = fluorodeoxyglucose positron-emission tomography; $\mathrm{H}$ = full field digital mammography; $\mathrm{I}$ = handheld breast ultrasound; $\mathrm{J}$ = magnetic resonance imaging; $\mathrm{K}$ = automated breast volume scanner; $\mathrm{L}=$ magnetic resonance mammography; $\mathrm{M}$ = scintimammography; $\mathrm{N}$ = single photon emission computed tomography scintimammography; $\mathrm{O}=$ ultrasound elastography; $\mathrm{P}=$ ultrasonography; $\mathrm{Q}=$ mammography+ultrasonography; $\mathrm{R}=$ mammography + scintimammography; $\mathrm{S}=$ ultrasound elastography + ultrasonography; OR = Odds ratios; 95\% CI = 95\% confidence intervals; PPV = positive predictive value; NPV = negative predictive value. The terms with lines represent statistical differences. If $95 \%$ confidence interval does not contain 1 , there are statistical differences

imaging; $\mathrm{K}$ = automated breast volume scanner; $\mathrm{L}=$ magnetic resonance mammography; $\mathrm{M}=$ scintimammography; $\mathrm{N}$ = single photon emission computed tomography scintimammography; 0 = ultrasound elastography; $\mathrm{P}=$ ultrasonography; $\mathrm{Q}$ = mammography + ultrasonography; $\mathrm{R}=$ mammography + scintimammography; and S = ultrasound elastography + ultrasonography. According to Fig. 3, it is revealed that the $\mathrm{MG}$ and US methods diagnosed the highest number of $\mathrm{BC}$ patients and have relatively more studies in terms of sensitivity, specificity, PPV, NPV and accuracy.

Inconsistency tests of the sensitivity, specificity, PPV, NPV and accuracy of 19 imaging methods for $B C$

The sensitivity, specificity, PPV, NPV and accuracy of the included imaging methods were analyzed using inconsistency tests employing the node-splitting method, and the results indicated consistency among the direct and indirect evidence of all outcomes, and therefore, the consistency model was adopted in this study (all $P>0.05$ ) (Table 3 and 4 ).

\section{Main results of network meta-analysis of the diagnostic value of 19 imaging methods for} $B C$

The results of this network meta-analysis revealed that the sensitivity and NPV of the CE-MRI, MRM and MG + SMM methods were relatively higher compared with the MG

\begin{tabular}{|c|c|c|c|c|c|c|}
\hline \multirow{2}{*}{ Included study } & \multirow{2}{*}{ Comparison } & \multicolumn{2}{|c|}{ Heterogeneity test } & \multicolumn{3}{|c|}{ Pairwise meta-analysis } \\
\hline & & $I^{2}$ & $\mathrm{Ph}_{\mathrm{h}}$ & OR $(95 \% \mathrm{CI})$ & $\mathrm{z}$ & $\mathrm{P}$ \\
\hline \multicolumn{7}{|l|}{ Sensitivity } \\
\hline 3 studies & M vs. A & $0.0 \%$ & 0.919 & $0.89(0.51,1.54)$ & 0.42 & 0.673 \\
\hline 7 studies & Lvs. A & $64.3 \%$ & $\underline{0.01}$ & $4.00(2.72,5.89)$ & 7.04 & 0.000 \\
\hline 1 study & C vs. A & $\mathrm{NA}$ & $\mathrm{NA}$ & $0.79(0.33,1.86)$ & 0.55 & 0.585 \\
\hline 13 studies & Pvs. A & $75.3 \%$ & $\underline{0.000}$ & $1.84(1.52,2.23)$ & $\underline{6.30}$ & 0.000 \\
\hline 2 studies & Rvs. A & $0.00 \%$ & $\underline{0.592}$ & $3.28(1.48,7.26)$ & 2.93 & 0.003 \\
\hline 1 study & D vs. A & NA & $\mathrm{NA}$ & $2.65(0.89,7.92)$ & 1.75 & 0.081 \\
\hline 2 studies & Qvs. A & $69.0 \%$ & $\underline{0.073}$ & $3.97(2.34,6.74)$ & $\underline{5.10}$ & 0.000 \\
\hline 2 studies & O vs. A & $0.0 \%$ & 0.367 & $0.77(0.43,1.37)$ & 0.88 & 0.380 \\
\hline 2 studies & $\underline{S \text { vs. A }}$ & $\underline{0.0 \%}$ & $\underline{0.371}$ & $3.89(1.76,8.58)$ & $\underline{3.36}$ & 0.001 \\
\hline$\overline{1 \text { study }}$ & $\overline{\text { Lvs. A }}$ & $\overline{\mathrm{NA}}$ & $\overline{\mathrm{NA}}$ & $10.64(1.30,87.26)$ & $\overline{2.20}$ & 0.028 \\
\hline 2 studies & $\overline{\text { Evs. A }}$ & $70.2 \%$ & $0 . \overline{067}$ & $1.46(0.76,2.78)$ & $\overline{1.14}$ & 0.255 \\
\hline 1 study & B vs. A & $\underline{\mathrm{NA}}$ & $\underline{\mathrm{NA}}$ & $10.17(5.21,19.87)$ & 6.79 & 0.000 \\
\hline 1 study & $\overline{\mathrm{K} \text { vs. } \mathrm{A}}$ & $\overline{\mathrm{NA}}$ & $\overline{\mathrm{NA}}$ & $0.45(0.10,1.98)$ & $\overline{1.06}$ & 0.289 \\
\hline 1 study & Ivs. A & $\mathrm{NA}$ & NA & $7.71(0.38,155.64)$ & 1.33 & 0.183 \\
\hline \multicolumn{7}{|l|}{ Specificity } \\
\hline 3 studies & M vs. A & $56.6 \%$ & $\underline{0.100}$ & $4.72(2.24,9.92)$ & $\underline{4.09}$ & 0.000 \\
\hline$\overline{7 \text { studies }}$ & $\overline{\text { Jvs. A }}$ & $90.0 \%$ & $\overline{0.000}$ & $0.91(0.67,1.22)$ & 0.65 & 0.514 \\
\hline 1 study & C vs. A & NA & NA & $0.88(0.43,1.84)$ & 0.33 & 0.743 \\
\hline 13 studies & Pvs. A & $85.5 \%$ & $\underline{0.000}$ & $0.35(0.32,0.39)$ & 22.57 & 2.000 \\
\hline 2 studies & $\overline{\mathrm{R} \text { vs. A }}$ & $84.0 \%$ & $\overline{0.012}$ & $0.84(0.37,1.91)$ & 0.42 & 0.676 \\
\hline 1 study & D vs. A & NA & NA & $0.60(0.22,1.62)$ & 1.00 & 0.318 \\
\hline 2 studies & Qvs. A & $92.4 \%$ & $\underline{0.000}$ & $0.22(0.12,0.38)$ & $\underline{5.25}$ & 0.000 \\
\hline 2 studies & Ovs. A & $96.7 \%$ & 0.000 & $0.76(0.47,1.23)$ & 1.10 & 0.270 \\
\hline 2 studies & S vs. A & $97.4 \%$ & 0.000 & $0.64(0.40,1.01)$ & 1.90 & 0.058 \\
\hline 1 study & Lvs. A & NA & $\mathrm{NA}$ & $1.18(0.38,3.63)$ & 0.29 & 0.775 \\
\hline 2 studies & Evs. A & $0.0 \%$ & 0.616 & $0.89(0.55,1.43)$ & 0.49 & 0.624 \\
\hline 1 study & B vs. A & NA & NA & $0.47(0.14,1.64)$ & 1.18 & 0.237 \\
\hline 1 study & K vs. A & $\mathrm{NA}$ & NA & $2.98(0.79,11.25)$ & 1.61 & 0.107 \\
\hline 1 study & Ivs. A & $\mathrm{NA}$ & NA & $0.49(0.13,1.93)$ & 1.02 & 0.308 \\
\hline \multicolumn{7}{|l|}{ PPV } \\
\hline 3 studies & M vs. A & $\underline{0.0 \%}$ & $\underline{0.508}$ & $2.23(1.19,4.16)$ & $\underline{2.51}$ & 0.012 \\
\hline$\overline{7 \text { studies }}$ & $\overline{\text { Jvs. A }}$ & $\overline{78.1 \%}$ & $\overline{0.000}$ & $1.17(0.89,1.53)$ & $\overline{1.09}$ & $\overline{0.274}$ \\
\hline 1 study & Cvs. A & NA & $\mathrm{NA}$ & $0.77(0.37,1.59)$ & 0.71 & 0.476 \\
\hline 13 studies & P vs. A & $69.2 \%$ & $\underline{0.000}$ & $\underline{0.63(0.53,0.74)}$ & $\underline{5.39}$ & 0.000 \\
\hline 2 studies & $\overline{\mathrm{Rvs} . \mathrm{A}}$ & $74.7 \%$ & $\overline{0.047}$ & $1.06(0.54,2.08)$ & $\overline{0.16}$ & 0.874 \\
\hline 1 study & D vs. A & NA & NA & $0.84(0.38,1.87)$ & 0.42 & 0.677 \\
\hline 2 studies & Qvs. A & $71.9 \%$ & $\underline{0.059}$ & $0.58(0.37,0.91)$ & 2.37 & 0.018 \\
\hline 2 studies & $\overline{\text { Ovs. A }}$ & $\overline{94.5 \%}$ & $\overline{0.000}$ & $0.89(0.52,1.54)$ & $\overline{0.41}$ & 0.684 \\
\hline 2 studies & S vs. A & $95.1 \%$ & 0.000 & $1.05(0.62,1.78)$ & 0.16 & 0.869 \\
\hline 1 study & Lvs. A & NA & $\mathrm{NA}$ & $1.27(0.55,2.91)$ & 0.56 & 0.578 \\
\hline 2 studies & Evs. A & $0.0 \%$ & 0.824 & $1.14(0.73,1.79)$ & 0.58 & 0.564 \\
\hline 1 study & B vs. A & $\mathrm{NA}$ & $\mathrm{NA}$ & $0.86(0.25,2.94)$ & 0.24 & 0.810 \\
\hline 1 study & K vs. A & NA & NA & $1.64(0.53,5.07)$ & 0.86 & 0.387 \\
\hline 1 study & Ivs. A & NA & NA & $0.87(0.34,2.21)$ & 0.30 & 0.765 \\
\hline NPV & & & & & & \\
\hline 3 studies & M vs. A & $0.0 \%$ & 0.530 & $1.45(0.73,2.86)$ & 1.06 & 0.288 \\
\hline 7 studies & Lvs. A & $54.8 \%$ & $\underline{0.039}$ & $2.54(1.68,3.86)$ & $\underline{4.39}$ & 0.000 \\
\hline 1 study & C vs. A & $\mathrm{NA}$ & $\mathrm{NA}$ & $0.91(0.38,2.15)$ & 0.22 & 0.826 \\
\hline 13 studies & Pvs. A & $56.8 \%$ & 0.006 & $1.13(0.93,1.39)$ & 1.22 & 0.223 \\
\hline 2 studies & $\underline{R}$ vs. A & $43.5 \%$ & $\underline{0.183}$ & $2.72(1.08,6.84)$ & $\underline{2.13}$ & 0.033 \\
\hline$\overline{1 \text { study }}$ & $\overline{\text { D vs. A }}$ & $\mathrm{NA}$ & $\overline{\mathrm{NA}}$ & $1.89(0.55,6.57)$ & 1.01 & 0.314 \\
\hline 2 studies & Q vs. A & $0.0 \%$ & 0.674 & $1.28(0.66,2.50)$ & 0.74 & 0.461 \\
\hline 2 studies & O vs. A & $69.5 \%$ & 0.070 & $0.79(0.47,1.33)$ & 0.89 & 0.372 \\
\hline 2 studies & $\underline{S \text { vs. A }}$ & $\underline{0.0 \%}$ & $\underline{0.758}$ & $3.07(1.43,6.58)$ & 2.88 & 0.004 \\
\hline 1 study & Lvs. A & NA & $\underline{\mathrm{NA}}$ & $9.90(1.06,92.66)$ & 2.01 & 0.045 \\
\hline 2 studies & $\overline{\text { Evs. A }}$ & $5 \overline{3.9} \%$ & $0 . \overline{14} 1$ & $\overline{1.35(0.69,2.63)}$ & $\overline{0.88}$ & 0.381 \\
\hline 1 study & B vs. A & $\mathrm{NA}$ & $\mathrm{NA}$ & $5.58(2.78,11.21)$ & 4.84 & 0.000 \\
\hline 1 study & Kvs. A & $\mathrm{NA}$ & $\mathrm{NA}$ & $0.81(0.16,4.20)$ & 0.25 & 0.804 \\
\hline 1 study & I vs. A & NA & NA & $4.53(0.19,105.84)$ & 0.94 & 0.347 \\
\hline Accuracy & & & & & & \\
\hline 3 studies & M vs. A & $39.9 \%$ & $\underline{0.189}$ & $1.62(1.07,2.45)$ & $\underline{2.28}$ & 0.022 \\
\hline 7 studies & Lvs. A & $80.8 \%$ & 0.000 & $1.64(1.32,2.04)$ & 4.42 & 0.000 \\
\hline$\overline{1 \text { study }}$ & C vs. A & $\mathrm{NA}$ & $\overline{\mathrm{NA}}$ & $0.83(0.48,1.45)$ & $\overline{0.65}$ & $\overline{0.513}$ \\
\hline 13 studies & Pvs. A & $94.6 \%$ & $\underline{0.000}$ & $\underline{0.49(0.46,0.53)}$ & 17.42 & $2 \underline{0.000}$ \\
\hline 2 studies & $\overline{\mathrm{R} \text { vs. } \mathrm{A}}$ & $85.1 \%$ & $\overline{0.010}$ & $1.65(0.99,2.75)$ & $\overline{1.91}$ & 0.056 \\
\hline 1 study & D vs. A & NA & NA & $1.18(0.62,2.24)$ & 0.49 & 0.622 \\
\hline 2 studies & Q vs. A & $0.0 \%$ & 0.531 & $1.06(0.76,1.48)$ & 0.34 & 0.735 \\
\hline 2 studies & O vs. A & $95.9 \%$ & 0.000 & $0.77(0.54,1.11)$ & 1.38 & 0.167 \\
\hline 2 studies & S vs. A & $97.2 \%$ & 0.000 & $1.08(0.74,1.58)$ & 0.39 & 0.698 \\
\hline 1 study & L vs. A & $\mathrm{NA}$ & NA & $1.87(0.89,3.91)$ & 1.65 & 0.099 \\
\hline 2 studies & Evs. A & $51.1 \%$ & 0.153 & $1.05(0.73,1.52)$ & 0.28 & 0.778 \\
\hline 1 study & B vs. A & NA & $\underline{\mathrm{NA}}$ & $0.20(0.11,0.34)$ & $\underline{5.96}$ & 0.000 \\
\hline 1 study & $\overline{\mathrm{Kvs} . \mathrm{A}}$ & $\overline{\mathrm{NA}}$ & $\overline{\mathrm{NA}}$ & $1.23(0.50,3.00)$ & $\overline{0.45}$ & $\overline{0.650}$ \\
\hline 1 study & I vs. A & NA & NA & $1.00(0.42,2.39)$ & 0.00 & 1.000 \\
\hline
\end{tabular}


Fig. 2. Evaluation of quality assessment of diagnostic accuracy studies (QUADAS) of included studies. Note: Thirty-nine diagnostic tests were analyzed in this network meta-analysis.

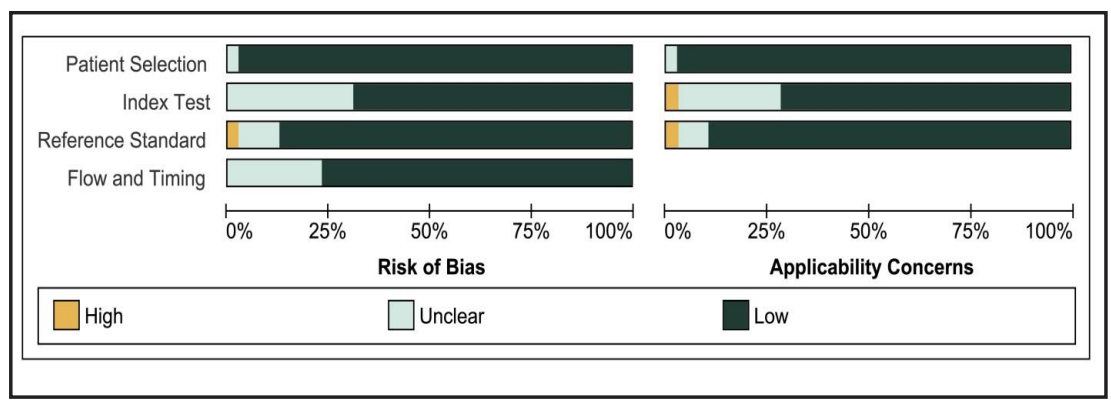

Fig. 3. Evidence network plot of diagnostic value of nineteen imaging methods for BC. Note: $A$ = mammography (MG); B = breast-specific gamma imaging (BSGI); $\mathrm{C}=$ color Doppler sonography (CD); $\mathrm{D}=$ contrastenhanced magnetic resonance imaging (CE-MRI); E = digital breast tomosynthesis (DBT); $\mathrm{F}$ = fluorodeoxyglucose positron-emission tomography/computed tomography (FDG PET/CT); G = fluorodeoxyglucose positron-emission tomography (FDG-PET); $\mathrm{H}=$ full field digital mammography (FFDM); I = handheld breast ultrasound (HHUS); J = magnetic resonance imaging (MRI); $\mathrm{K}$ = automated breast volume scanner (ABUS); $\mathrm{L}=$ magnetic resonance mammography (MRM); $\mathrm{M}$ = scintimammography (SMM); $\mathrm{N}$ = single photon emission computed tomography scintimammography (SPECT SMM); O = ultrasound elastography (UE);

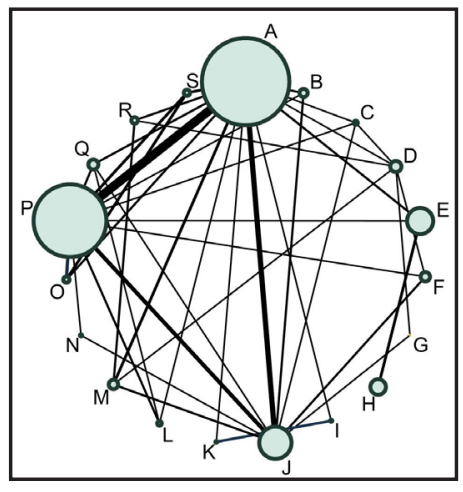
$\mathrm{P}=$ ultrasonography (US); $\mathrm{Q}=$ mammography + ultrasonography $(\mathrm{MG}+$ US); R = mammography + scintimammography (MG + SMM); S = ultrasound elastography + ultrasonography (UE + US); and BC = breast cancer.

Table 3. $P$ values of direct and indirect pairwise comparisons among nineteen diagnostic modalities under five outcome indicators. Note: PPV = positive predictive value; NPV = negative predictive value

\begin{tabular}{|c|c|c|c|c|c|c|c|c|c|c|c|c|c|c|c|}
\hline \multirow[b]{2}{*}{ Pairwise comparisons } & \multicolumn{15}{|c|}{$\mathrm{P}$ values } \\
\hline & B vs. A & Cvs. A & D vs. A & Evs. A & Jvs. A & Lvs. A & M vs. A & O vs. A & P vs. A & S vs. A & $\begin{array}{c}\text { J vs. } \\
\text { B }\end{array}$ & P vs. B & D vs. C & J vs. C & F vs. D \\
\hline Sensitivity & 0.16 & 0.068 & 0.11 & 0.71 & 0.62 & 0.69 & 0.46 & 0.11 & 0.076 & 0.12 & 0.45 & 0.97 & 0.082 & 0.051 & 0.099 \\
\hline Specificity & 0.20 & 0.35 & 0.68 & 0.75 & 0.32 & 0.30 & 0.81 & 0.85 & 0.53 & 0.65 & 0.34 & 0.62 & 0.32 & 0.48 & 0.068 \\
\hline PPV & 0.15 & 0.16 & 0.60 & 0.82 & 0.38 & 0.50 & 0.95 & 0.46 & 0.71 & 0.54 & 0.38 & 0.75 & 0.14 & 0.22 & 0.082 \\
\hline NPV & 0.80 & 0.10 & 0.08 & 0.45 & 0.63 & 0.87 & 0.51 & 0.06 & 0.77 & 0.23 & 0.97 & 0.70 & 0.13 & 0.05 & 0.079 \\
\hline Accuracy & 0.51 & 0.32 & 0.93 & 0.89 & 0.45 & 0.93 & 0.94 & 0.14 & 0.78 & 0.31 & 0.28 & 0.18 & 0.30 & 0.59 & 0.76 \\
\hline
\end{tabular}

Table 4. $P$ values of direct and indirect pairwise comparisons among nineteen diagnostic modalities under five outcome indicators. Note: PPV = positive predictive value; NPV = negative predictive value

\begin{tabular}{|c|c|c|c|c|c|c|c|c|c|c|c|c|c|}
\hline \multirow{2}{*}{ Pairwise comparisons } & \multicolumn{13}{|c|}{$P$ values } \\
\hline & G vs. D & M vs. D & R vs. D & P vs. E & EJ vs. F & Pvs. F & J vs. G & M vs. J & I N vs. J & JP vs. J & Q vs. J & Q vs. L & P vs. N \\
\hline Sensitivity & 0.11 & 0.12 & 0.14 & 0.69 & 0.18 & 0.16 & 0.11 & 0.68 & 0.65 & 0.35 & 0.91 & 0.99 & 0.66 \\
\hline Specificity & 0.32 & 0.97 & 0.50 & 0.75 & 0.06 & 0.068 & 0.333 & 0.98 & 0.46 & 0.73 & 0.63 & 0.68 & 0.44 \\
\hline PPV & 0.42 & 0.96 & 0.40 & 0.81 & 0.06 & 0.054 & 0.42 & 0.98 & 0.32 & 0.76 & 0.52 & 0.74 & 0.33 \\
\hline NPV & 0.05 & 0.06 & 0.05 & 0.44 & 0.13 & 0.47 & 0.07 & 0.37 & 0.52 & 0.63 & 0.60 & 0.87 & 0.50 \\
\hline Accuracy & 0.51 & 0.65 & 0.18 & 0.88 & 0.77 & 0.81 & 0.51 & 0.36 & 0.51 & 0.61 & 0.63 & 0.88 & 0.53 \\
\hline
\end{tabular}

method. In addition, the sensitivity, NPV and accuracy of the MRI method were relatively higher compared to the MG method. The BSGI method exhibited relatively higher PPV and accuracy, the FDG PET/CT method exhibited higher specificity and PPV, and the sensitivity of UE + US method ranked higher compared to the MG method (Fig. 4 and see online suppl. material, Suppl. Tables 1-5).

SUCRA values of the diagnostic value of 19 imaging methods for $B C$

As shown in Table 5, the SUCRA values of the diagnostic value of 19 imaging methods for BC indicated that the MRM method ranked the highest in terms of sensitivity and NPV 
Table 5. SUCRA values of nineteen diagnostic modalities under five outcome indicators. Note: SUCRA = surface under the cumulative ranking curves; $\mathrm{A}=$ mammography; $\mathrm{B}=$ breast-specific gamma imaging; $\mathrm{C}=$ Color Doppler sonography; $\mathrm{D}=$ contrast-enhanced magnetic resonance imaging; $\mathrm{E}=$ digital breast tomosynthesis; $\mathrm{F}$ = fluorodeoxyglucose positron-emission tomography/computed tomography; $\mathrm{G}=$ fluorodeoxyglucose positron-emission tomography; $\mathrm{H}=$ full field digital mammography; I = handheld breast ultrasound; $\mathrm{J}$ = magnetic resonance imaging; $\mathrm{K}=$ automated breast volume scanner; $\mathrm{L}=$ magnetic resonance mammography; $\mathrm{M}=$ scintimammography; $\mathrm{N}$ = single photon emission computed tomography scintimammography; $\mathrm{O}=$ ultrasound elastography; $\mathrm{P}=$ ultrasonography; $\mathrm{Q}=$ mammography + ultrasonography; $\mathrm{R}$ = mammography + scintimammography; $\mathrm{S}=$ ultrasound elastography + ultrasonography; PPV = positive predictive value; NPV = negative predictive value. The Figures with underlines represent the largest SUCRA values.

\begin{tabular}{lccccc}
\hline \multirow{2}{*}{ Treatment } & \multicolumn{5}{c}{ SUCRA value } \\
& Sensitivity & Specificity & PPV & NPV & Accuracy \\
\hline A & 0.3030 & 0.5132 & 0.41530 .2726 & 0.3705 \\
$\mathrm{~B}$ & 0.6620 & 0.7763 & 0.83790 .6937 & 0.9742 \\
$\mathrm{C}$ & 0.2670 & 0.6153 & 0.52680 .2716 & 0.4721 \\
$\mathrm{D}$ & 0.8830 & 0.5842 & 0.57890 .8721 & 0.5479 \\
$\mathrm{E}$ & 0.3670 & 0.4847 & 0.46420 .4837 & 0.4463 \\
$\mathrm{~F}$ & 0.1160 & $\underline{0.9437}$ & $\underline{0.9495} 0.1911$ & 0.4600 \\
$\mathrm{G}$ & 0.7800 & 0.3584 & 0.3158 & 0.7758 & 0.3463 \\
$\mathrm{H}$ & 0.3160 & 0.4432 & 0.42110 .3832 & 0.3858 \\
$\mathrm{I}$ & 0.5560 & 0.4821 & 0.38050 .5216 & 0.4063 \\
$\mathrm{~J}$ & 0.7470 & 0.4742 & 0.57530 .7632 & 0.7084 \\
$\mathrm{~K}$ & 0.3180 & 0.5958 & 0.62210 .3947 & 0.5432 \\
$\mathrm{~L}$ & 0.9520 & 0.2095 & 0.2800 & 0.9011 & 0.6179 \\
$\mathrm{M}$ & 0.3950 & 0.8326 & 0.8079 & 0.5811 & 0.6726 \\
$\mathrm{~N}$ & 0.3680 & 0.6384 & 0.6242 & 0.4368 & 0.6132 \\
$\mathrm{O}$ & 0.3390 & 0.4458 & 0.45530 .2816 & 0.3800 \\
$\mathrm{P}$ & 0.4710 & 0.2868 & 0.30260 .3537 & 0.3158 \\
$\mathrm{Q}$ & 0.6370 & 0.2511 & 0.2784 & 0.4284 & 0.4426 \\
$\mathrm{R}$ & 0.7580 & 0.6353 & 0.62950 .7505 & 0.7658 \\
S & 0.7040 & 0.4221 & 0.5142 & 0.6200 & 0.5126 \\
\hline
\end{tabular}

Fig. 4. Forest plots of diagnostic value of nineteen imaging methods for BC. Note: $\mathrm{A}=$ mammography (MG); $\mathrm{B}=$ breast-specific gamma imaging (BSGI); $\mathrm{C}$ = color Doppler sonography (CD); $\mathrm{D}=$ contrast-enhanced magnetic resonance imaging (CE-MRI); $\mathrm{E}=$ digital breast tomosynthesis (DBT); F = fluorodeoxyglucose positron-emission tomography/computed tomography (FDG PET/CT); G = fluorodeoxyglucose positron-emission tomography (FDG-PET); $\mathrm{H}$ = full field digital mammography (FFDM); I = handheld breast ultrasound (HHUS); J = magnetic resonance imaging (MRI); $\mathrm{K}$ = automated breast volume scanner (ABUS); L = magnetic resonance mammography (MRM); $\mathrm{M}$ = scintimammography (SMM); $\mathrm{N}=$ single photon emission computed tomography scintimammography (SPECT SMM); $0=$ ultrasound elastography (UE); P = ultrasonography (US); Q = mammography + ultrasonography $(M G+U S) ; R$ = mammography + scintimammography (MG + SMM); S = ultrasound elastography + ultrasonography (UE + US); and $\mathrm{BC}=$ breast cancer.

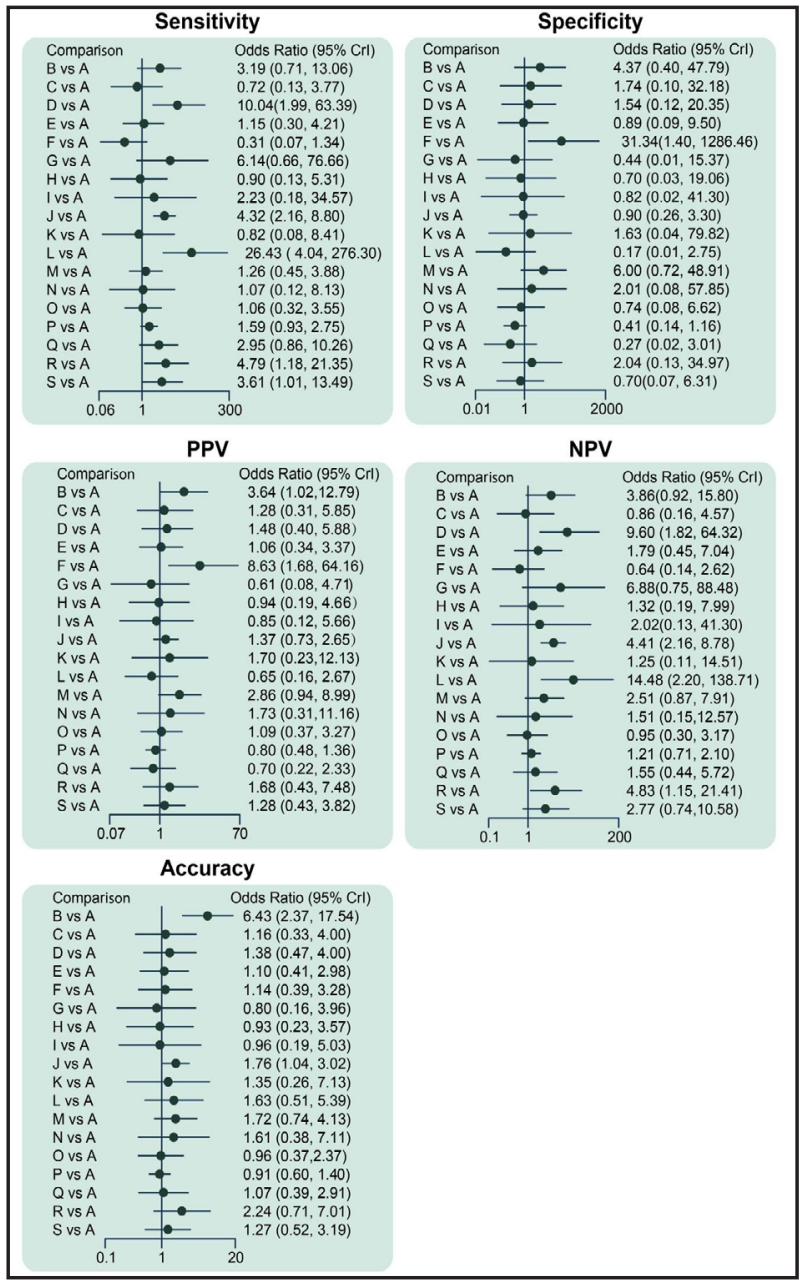

(sensitivity: 95.2\%; NPV: 90.1\%), while the FDG PET/CT method exhibited the highest sensitivity and PPV (specificity: 94.37\%; PPV: 94.95\%), and the BSGI method exhibited the highest accuracy among all 19 imaging methods (97.42\%). 
Fig. 5. Cluster ranking plots based on SUCRA values of sensitivity, specificity and accuracy of nineteen imaging methods for BC. Note: $\mathrm{A}=$ mammography (MG); $\mathrm{B}=$ breast-specific gamma imaging (BSGI); $\mathrm{C}=$ Color Doppler sonography (CD); $\mathrm{D}=$ contrast-enhanced magnetic resonance imaging (CE-MRI); $\mathrm{E}=$ digital breast tomosynthesis (DBT); $\mathrm{F}=$ fluorodeoxyglucose positron-emission tomography/ computed tomography (FDG PET/CT); G = fluorodeoxy-

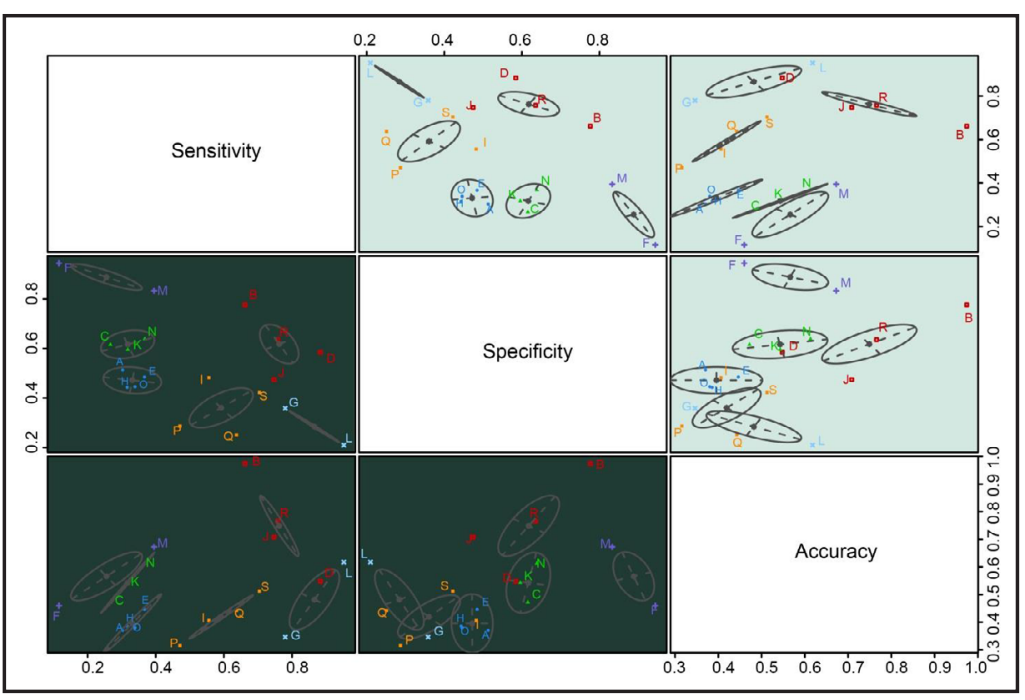
glucose positron-emission tomography (FDG-PET); H = full field digital mammography (FFDM); I = handheld breast ultrasound (HHUS); $\mathrm{J}=$ magnetic resonance imaging (MRI); $\mathrm{K}$ = automated breast volume scanner (ABUS); $\mathrm{L}=$ magnetic resonance mammography (MRM); $\mathrm{M}$ = scintimammography (SMM); $\mathrm{N}$ = single photon emission computed tomography scintimammography (SPECT SMM); $\mathrm{O}=$ ultrasound elastography (UE); $\mathrm{P}=$ ultrasonography (US); Q = mammography + ultrasonography (MG + US); R = mammography + scintimammography (MG + $\mathrm{SMM}) ; \mathrm{S}=$ ultrasound elastography + ultrasonography (UE + US); BC = breast cancer; and SUCRA = surface under the cumulative ranking.

Fig. 6. Comparison-adjusted funnel plot for diagnostic value of nineteen imaging methods for $\mathrm{BC}$ Note: the red line shows the null hypothesis that the study-specific effect sizes are not distinct from the respective comparison-specific pooled effect estimates; distinct colors suggest distinct comparisons; net funnel adds linear regression line including the study effect sizes centered at the comparisonspecific summary effect $\left(\mathrm{y}_{\mathrm{IXY}}-\mu_{\mathrm{XY}}\right)$; $\mathrm{y}_{\mathrm{iXY}}$ means effect value of a single study, and $\mu_{\mathrm{XY}}$ means mean effect value of all studies relative to the control group; $\mathrm{A}=$ mammography
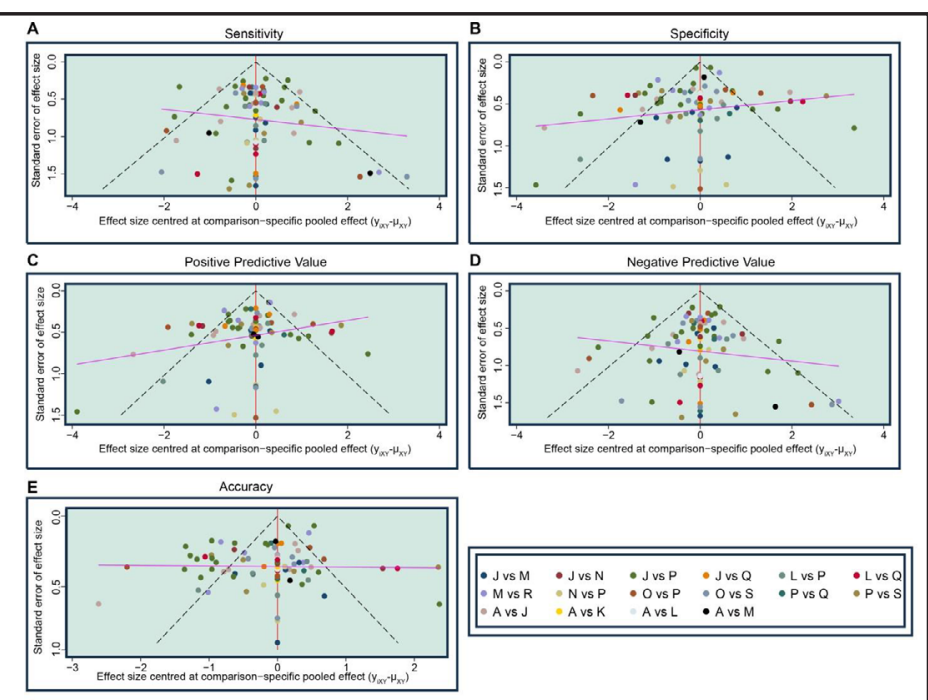
(MG); $\mathrm{B}=$ breast-specific gamma imaging (BSGI); $\mathrm{C}=$ Color Doppler sonography (CD); D = contrast-enhanced magnetic resonance imaging (CE-MRI); E = digital breast tomosynthesis (DBT); F = fluorodeoxyglucose positron-emission tomography/ computed tomography (FDG PET/CT); G = fluorodeoxyglucose positron-emission tomography (FDG-PET); $\mathrm{H}$ = full field digital mammography (FFDM); I = handheld breast ultrasound (HHUS); J = magnetic resonance imaging (MRI); $\mathrm{K}$ = automated breast volume scanner (ABUS); L = magnetic resonance mammography (MRM); $\mathrm{M}$ = scintimammography (SMM); $\mathrm{N}$ = single photon emission computed tomography scintimammography (SPECT SMM); O = ultrasound elastography (UE); P = ultrasonography (US); Q = mammography + ultrasonography (MG + US); R = mammography + scintimammography (MG + SMM); S = ultrasound elastography + ultrasonography (UE + US); BC = breast cancer.

\section{KARGER}


Cluster analysis of the diagnostic value of 19 imaging methods for $B C$

The cluster analysis based on SUCRA values revealed that the BSGI, MG + SMM, MRI and CE-MRI methods showed the greatest diagnostic value in $B C$ detection, with reference to sensitivity, specificity and accuracy (Fig. 5).

Assessment of publication bias of the diagnostic value of 19 imaging methods for $B C$

The results of assessment of publication bias showed symmetrical distribution, indicating no small sample effect or publication bias in this network meta-analysis (Fig. 6).

\section{Discussion}

$\mathrm{BC}$ is the leading cancer among women worldwide. Early detection of BC plays a critical role in improving the quality of life and survival of patients suffering from $\mathrm{BC}$ [72]. Women presenting with symptoms or palpable findings during clinical examinations are usually investigated with breast imaging, which generally consists of the MG method, breast sonography, or both [56]. A study found that consecutive patients could reduce the selection bias [73]. This study performed an NMA with consecutive patients and found that the diagnostic value of the BSGI, MG + SMM, MRI and CE-MRI methods for BC was relatively higher in terms of sensitivity, specificity and accuracy.

This NMA showed that compared with the MG method, the CE-MRI, MRI, MRM, MG + SMM and UE + US methods were relatively more sensitive; the FDG PET/CT method exhibited higher specificity; and the BSGI and MRI methods were more accurate. A metaanalysis of diagnostic tests serves as a useful way to summarize findings by analyzing different studies, and a previously conducted meta-analysis comparing the diagnostic value of FDG-PET, MRI, and bone scintigraphy (BS) in the detection of bone metastases of BC found that MRI was a superior imaging method in comparison to FDG-PET and BS [74]. Biopsy is a common method in diagnosis of BC [75-77], which is consistent with our findings (Table 1). In addition, another study found that vacuum-assisted breast biopsy had high levels of diagnostic accuracy and sensitivity when evaluating mammographically detected breast by performing a meta-analysis [78]. A screening MG was used to examine the breasts of an asymptomatic woman, while a diagnostic MG was performed in a patient presenting with signs or symptoms of breast disease: a possible abnormality detected on screening MG or other imaging, or prior MG findings requiring imaging follow up [79]. The factors implicated in missing identification of cancers include technique sensitivity, distracting lesions, tumor growth rate, tumor growth pattern and the background upon which the tumor is displayed. The background in the MG method consists of normal dense breast structures superimposed onto a two-dimensional (2D) plane, comprising a so-called "anatomic background", which plays a larger role than quantum noise in the non-detection of certain lesion types and sizes [80]. The sensitivity of the MG method is limited in dense breast tissue, due to the presence of overlapping fibroglandular tissue, which reduces the conspicuousness of abnormalities [81]. Tumor growth pattern is an additional factor influencing cancer visibility in breast tissues. Tumors that do not produce a mass are often difficult to detect using the MG method [80].

The results of cluster analysis indicated that the BSGI, MG + SMM, MRI and CE-MRI methods showed the greatest diagnostic value in BC detection, in terms of sensitivity, specificity and accuracy. Compared with MG method, the BSGI method was comparatively more sensitive but at the cost of accuracy; the MRI method exhibited higher sensitivity and accuracy; the UE + US and MRM methods were more sensitive; the SMM method exhibited relatively higher specificity and accuracy; the US method exhibited lower specificity and accuracy but higher sensitivity; the MG + US method exhibited higher specificity and lower sensitivity; and the NPVMG + SMM method had higher sensitivity. Although, the combination of MG, clinical examination, and MRI methods was more sensitive than any other individual or combination tests, the sensitivity of the MG method ranges from $30 \%$ to $98 \%$ and has been reported to be as low as 30\%-48\% in the cases of dense breasts [57]. Along with 
MG and ultrasound, MRI is the gold standard in diagnosing breast imaging; however, it is a complicated and expensive procedure with relatively low specificity, which leads to high false-positive rates [82]. The BSGI method has an equally high sensitivity, but it is more specific and much easier to interpret, therefore exhibiting great potential to indicate or exclude the extent of BC [83]. Moreover, according to a recent meta-analysis, MRI significantly boosted mastectomy rates and was suggested to play an unfavorable harm-benefit ratio for routine use of MRI preoperation in BC patients [84]. When comparing SMM and MG, the SMM method exhibited better diagnostic accuracy in the detection of recurrence of BC [85]. SMM is often considered to be a supplementary tool in the preoperative work of BC patients, and the high negative predictive value of SMM adds to its ability in the aspect of decreasing the amount of unnecessary surgical interventions or negative breast biopsies [86]. The CEMRI method is conducive to predicting the efficiency of neoadjuvant chemotherapy in BC and was, therefore, endowed with the potential to supply functional parameters that can be integrated to optimize the strategies for neoadjuvant chemotherapy [87]. Comparing the results of the SMM, MG and MRI methods, all three methods exhibited high sensitivity for the detection of palpable BC, but the SMM method presented with a significantly higher specificity in comparison to the MG and MRI methods [71].

This study could not offer enough direct comparisons of individual imaging method due to limited references and data, and the vast majority of the studies were of low quality, but we comprehensively compared the different diagnostic value and limitations of 19 imaging methods for BC. Additionally, the Chinese literature was not retrieved because of failure to conform to the QUADAS-2 quality standard, which might affect the final results of this network meta-analysis to some extent. In conclusion, the BSGI, MG + SMM, MRI and CE-MRI methods exhibited a higher diagnostic value for $\mathrm{BC}$, which provides evidence toward the further development of the diagnosis of $\mathrm{BC}$ to improve the survival of patients suffering from $\mathrm{BC}$.

\section{Acknowledgements}

We would like to acknowledge the helpful comments on this paper that were received from our reviewers.

\section{Disclosure Statement}

No conflict of interests exists.

\section{References}

1 Jia M, Zheng R, Zhang S, Zeng H, Zou X, Chen W: Female breast cancer incidence and mortality in 2011, China. J Thorac Dis 2015;7:1221-1226.

2 Siegel R, Naishadham D, Jemal A: Cancer statistics, 2012. CA Cancer J Clin 2012;62:10-29.

3 Diaby V, Tawk R, Sanogo V, Xiao H, Montero AJ: A review of systematic reviews of the cost-effectiveness of hormone therapy, chemotherapy, and targeted therapy for breast cancer. Breast Cancer Res Treat 2015;151:27-40.

4 Colombo PE, Milanezi F, Weigelt B, Reis-Filho JS: Microarrays in the 2010s: the contribution of microarraybased gene expression profiling to breast cancer classification, prognostication and prediction. Breast Cancer Res 2011;13:212.

5 Pracella D, Bonin S, Barbazza R, Sapino A, Castellano I, Sulfaro S, Stanta G: Are breast cancer molecular classes predictive of survival in patients with long follow-up? Dis Markers 2013;35:595-605.

6 Iorio MV, Casalini P, Piovan C, Braccioli L, Tagliabue E: Breast cancer and microRNAs: therapeutic impact. Breast 2011;20:S63-70.

7 Dumalaon-Canaria JA, Hutchinson AD, Prichard I, Wilson C: What causes breast cancer? A systematic review of causal attributions among breast cancer survivors and how these compare to expert-endorsed risk factors. Cancer Causes Control 2014;25:771-785. 


\section{Cellular Physiology Cell Physiol Biochem 2018;46:2041-2055 \begin{tabular}{l|l} 
and Biochemistry Published onIne: Vay 03, 2018 & $\begin{array}{l}\text { C } 2018 \text { The Author(s). Published by S. Karger AG, Basel } \\
\text { www.karger.com/cpb }\end{array}$
\end{tabular}}

Zhang et al.: Diagnostic Value of Imaging Methods for BC

8 Rakha EA: Pitfalls in outcome prediction of breast cancer. J Clin Pathol 2013;66:458-464.

9 Khan S, Bennit HF, Turay D, Perez M, Mirshahidi S, Yuan Y, Wall NR: Early diagnostic value of survivin and its alternative splice variants in breast cancer. BMC Cancer 2014;14:176.

10 Yersal 0, Barutca S: Biological subtypes of breast cancer: Prognostic and therapeutic implications. World J Clin Oncol 2014;5:412-424.

11 Bidlek M, Kovacs E, Feher K, Godeny M: [New trends and novel possibilities in the diagnostic imaging of breast cancer]. Magy Onkol 2015;59:44-55.

12 Weinstein S, Rosen M: Breast MR imaging: current indications and advanced imaging techniques. Radiol Clin North Am 2010;48:1013-1042.

13 Gruber IV, Rueckert M, Kagan KO, Staebler A, Siegmann KC, Hartkopf A, Wallwiener D, Hahn M: Measurement of tumour size with mammography, sonography and magnetic resonance imaging as compared to histological tumour size in primary breast cancer. BMC Cancer 2013;13:328.

14 Liang L, Yue Z, Du W, Li Y, Tao H, Wang D, Wang R, Huang Z, He N, Xie X, Han Z, Liu N, Li Z: Molecular Imaging of Inducible VEGF Expression and Tumor Progression in a Breast Cancer Model. Cell Physiol Biochem 2017;42:407-415.

15 Behrendt CE, Tumyan L, Gonser L, Shaw SL, Vora L, Paz IB, Ellenhorn JD, Yim JH: Evaluation of expert criteria for preoperative magnetic resonance imaging of newly diagnosed breast cancer. Breast 2014;23:341-345.

16 Mann RM, Kuhl CK, Kinkel K, Boetes C: Breast MRI: guidelines from the European Society of Breast Imaging. Eur Radiol 2008;18:1307-1318.

17 Brennan ME, Houssami N, Lord S, Macaskill P, Irwig L, Dixon JM, Warren RM, Ciatto S: Magnetic resonance imaging screening of the contralateral breast in women with newly diagnosed breast cancer: systematic review and meta-analysis of incremental cancer detection and impact on surgical management. J Clin Oncol 2009;27:5640-5649.

18 Turnbull L, Brown S, Harvey I, Olivier C, Drew P, Napp V, Hanby A, Brown J: Comparative effectiveness of MRI in breast cancer (COMICE) trial: a randomised controlled trial. Lancet 2010;375:563-571.

19 Peters NH, van Esser S, van den Bosch MA, Storm RK, Plaisier PW, van Dalen T, Diepstraten SC, Weits T, Westenend PJ, Stapper G, Fernandez-Gallardo MA, Borel Rinkes IH, van Hillegersberg R, Mali WP, Peeters PH: Preoperative MRI and surgical management in patients with nonpalpable breast cancer: the MONET randomised controlled trial. Eur J Cancer 2011;47:879-886.

20 Chang HT, Hu C, Chiu YL, Peng NJ, Liu RS: Role of 2-[18F] fluoro-2-deoxy-D-glucose-positron emission tomography/computed tomography in the post-therapy surveillance of breast cancer. PLoS One 2014;9:e115127.

-21 Salanti G: Indirect and mixed-treatment comparison, network, or multiple-treatments meta-analysis: many names, many benefits, many concerns for the next generation evidence synthesis tool. Res Synth Methods 2012;3:80-97.

22 Lumley T: Network meta-analysis for indirect treatment comparisons. Stat Med 2002;21:2313-2324.

23 Dias S, Welton NJ, Sutton AJ, Ades A: NICE DSU Technical Support Document 2: A Generalised Linear Modelling Framework for Pairwise and Network Meta-Analysis of Randomised Controlled Trials. 2011 last updated April 2014;

24 van Valkenhoef G, Dias S, Ades AE, Welton NJ: Automated generation of node-splitting models for assessment of inconsistency in network meta-analysis. Res Synth Methods 2016;7:80-93.

25 Hutton B, Salanti G, Caldwell DM, Chaimani A, Schmid CH, Cameron C, Ioannidis JP, Straus S, Thorlund K, Jansen JP, Mulrow C, Catala-Lopez F, Gotzsche PC, Dickersin K, Boutron I, Altman DG, Moher D: The PRISMA extension statement for reporting of systematic reviews incorporating network meta-analyses of health care interventions: checklist and explanations. Ann Intern Med 2015;162:777-784.

26 Whiting PF, Rutjes AW, Westwood ME, Mallett S, Deeks JJ, Reitsma JB, Leeflang MM, Sterne JA, Bossuyt PM, Group Q-: QUADAS-2: a revised tool for the quality assessment of diagnostic accuracy studies. Ann Intern Med 2011;155:529-536.

27 Chen LX, Li YL, Ning GZ, Li Y, Wu QL, Guo JX, Shi HY, Wang XB, Zhou Y, Feng SQ: Comparative efficacy and tolerability of three treatments in old people with osteoporotic vertebral compression fracture: a network meta-analysis and systematic review. PLoS One 2015;10:e0123153.

28 Tu YK, Needleman I, Chambrone L, Lu HK, Faggion CM, Jr.: A Bayesian network meta-analysis on comparisons of enamel matrix derivatives, guided tissue regeneration and their combination therapies. J Clin Periodontol 2012;39:303-314. 


\section{Cellular Physiology Cell Physiol Biochem 2018;46:2041-2055 \begin{tabular}{ll|l} 
and Biochemistry Published online: May 03, 2018 & $\begin{array}{l}\text { C) } 2018 \text { The Author(s). Published by S. Karger AG, Basel } \\
\text { www.karger.com/cpb }\end{array}$ \\
\hline
\end{tabular}}

-29 Zhu GQ, Shi KQ, Huang S, Wang LR, Lin YQ, Huang GQ, Chen YP, Braddock M, Zheng MH: Systematic review with network meta-analysis: the comparative effectiveness and safety of interventions in patients with overt hepatic encephalopathy. Aliment Pharmacol Ther 2015;41:624-635.

30 Chaimani A, Higgins JP, Mavridis D, Spyridonos P, Salanti G: Graphical tools for network meta-analysis in STATA. PLoS One 2013;8:e76654.

-31 Salanti G, Ades AE, Ioannidis JP: Graphical methods and numerical summaries for presenting results from multiple-treatment meta-analysis: an overview and tutorial. J Clin Epidemiol 2011;64:163-171.

-32 Mavridis D, Giannatsi M, Cipriani A, Salanti G: A primer on network meta-analysis with emphasis on mental health. Evid Based Ment Health 2015;18:40-46.

33 Moon HJ, Jung I, Park SJ, Kim MJ, Youk JH, Kim EK: Comparison of Cancer Yields and Diagnostic Performance of Screening Mammography vs. Supplemental Screening Ultrasound in 4394 Women with Average Risk for Breast Cancer. Ultraschall Med 2015;36:255-263.

-34 Magometschnigg HF, Baltzer PA, Fueger B, Helbich TH, Karanikas G, Dubsky P, Rudas M, Weber M, Pinker K: Diagnostic accuracy of (18)F-FDG PET/CT compared with that of contrast-enhanced MRI of the breast at 3 T. Eur J Nucl Med Mol Imaging 2015;42:1656-1665.

-35 Kim SA, Chang JM, Cho N, Yi A, Moon WK: Characterization of breast lesions: comparison of digital breast tomosynthesis and ultrasonography. Korean J Radiol 2015;16:229-238.

-36 Chou CP, Peng NJ, Chang TH, Yang TL, Hu C, Lin HS, Huang JS, Pan HB: Clinical roles of breast 3T MRI, FDG PET/CT, and breast ultrasound for asymptomatic women with an abnormal screening mammogram. J Chin Med Assoc 2015;78:719-725.

37 Grigoryev M, Thomas A, Plath L, Durmus T, Slowinski T, Diekmann F, Fischer T: Detection of microcalcifications in women with dense breasts and hypoechoic focal lesions: comparison of mammography and ultrasound. Ultraschall Med 2014;35:554-560.

38 Choi WJ, Cha JH, Kim HH, Shin HJ, Kim H, Chae EY, Hong MJ: Comparison of automated breast volume scanning and hand- held ultrasound in the detection of breast cancer: an analysis of 5, 566 patient evaluations. Asian Pac J Cancer Prev 2014;15:9101-9105.

-39 Thibault F, Dromain C, Breucq C, Balleyguier CS, Malhaire C, Steyaert L, Tardivon A, Baldan E, Drevon H: Digital breast tomosynthesis versus mammography and breast ultrasound: a multireader performance study. Eur Radiol 2013;23:2441-2449.

40 Shao H, Li B, Zhang X, Xiong Z, Liu Y, Tang G: Comparison of the diagnostic efficiency for breast cancer in Chinese women using mammography, ultrasound, MRI, and different combinations of these imaging modalities. J Xray Sci Technol 2013;21:283-292.

41 Golatta M, Franz D, Harcos A, Junkermann H, Rauch G, Scharf A, Schuetz F, Sohn C, Heil J: Interobserver reliability of automated breast volume scanner (ABVS) interpretation and agreement of ABVS findings with hand held breast ultrasound (HHUS), mammography and pathology results. Eur J Radiol 2013;82:e332336.

-42 Svahn TM, Chakraborty DP, Ikeda D, Zackrisson S, Do Y, Mattsson S, Andersson I: Breast tomosynthesis and digital mammography: a comparison of diagnostic accuracy. Br J Radiol 2012;85:e1074-1082.

43 Michell MJ, Iqbal A, Wasan RK, Evans DR, Peacock C, Lawinski CP, Douiri A, Wilson R, Whelehan P: A comparison of the accuracy of film-screen mammography, full-field digital mammography, and digital breast tomosynthesis. Clin Radiol 2012;67:976-981.

44 Kim BS, Moon BI, Cha ES: A comparative study of breast-specific gamma imaging with the conventional imaging modality in breast cancer patients with dense breasts. Ann Nucl Med 2012;26:823-829.

45 Kim BS: Usefulness of breast-specific gamma imaging as an adjunct modality in breast cancer patients with dense breast: a comparative study with MRI. Ann Nucl Med 2012;26:131-137.

46 Svane G, Azavedo E, Lindman K, Urech M, Nilsson J, Weber N, Lindqvist L, Ullberg C: Clinical experience of photon counting breast tomosynthesis: comparison with traditional mammography. Acta Radiol 2011;52:134-142.

47 Spangler ML, Zuley ML, Sumkin JH, Abrams G, Ganott MA, Hakim C, Perrin R, Chough DM, Shah R, Gur D: Detection and classification of calcifications on digital breast tomosynthesis and 2D digital mammography: a comparison. AJR Am J Roentgenol 2011;196:320-324.

48 Liew PL, Liu TJ, Hsieh MC, Lin HP, Lu CF, Yao MS, Chen CL: Rapid staining and immediate interpretation of fine-needle aspiration cytology for palpable breast lesions: diagnostic accuracy, mammographic, ultrasonographic and histopathologic correlations. Acta Cytol 2011;55:30-37. 


\section{Cellular Physiology Cell Physiol Biochem 2018;46:2041-2055

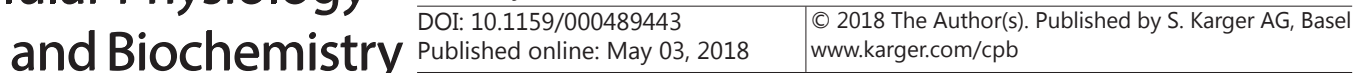

49 Moy L, Noz ME, Maguire GQ Jr., Melsaether A, Deans AE, Murphy-Walcott AD, Ponzo F: Role of fusion of prone FDG-PET and magnetic resonance imaging of the breasts in the evaluation of breast cancer. Breast J 2010;16:369-376.

-50 Leong LC, Sim LS, Lee YS, Ng FC, Wan CM, Fook-Chong SM, Jara-Lazaro AR, Tan PH: A prospective study to compare the diagnostic performance of breast elastography versus conventional breast ultrasound. Clin Radiol 2010;65:887-894.

-51 Hatzung G, Grunwald S, Zygmunt M, Geaid AA, Behrndt PO, Isermann R, Kohlmann T, Ohlinger R: Sonoelastography in the diagnosis of malignant and benign breast lesions: initial clinical experiences. Ultraschall Med 2010;31:596-603.

52 Benndorf M, Baltzer PA, Vag T, Gajda M, Runnebaum IB, Kaiser WA: Breast MRI as an adjunct to mammography: Does it really suffer from low specificity? A retrospective analysis stratified by mammographic BI-RADS classes. Acta Radiol 2010;51:715-721.

53 Vassiou K, Kanavou T, Vlychou M, Poultsidi A, Athanasiou E, Arvanitis DL, Fezoulidis IV: Characterization of breast lesions with CE-MR multimodal morphological and kinetic analysis: comparison with conventional mammography and high-resolution ultrasound. Eur J Radiol 2009;70:69-76.

54 Pediconi F, Catalano C, Roselli A, Dominelli V, Cagioli S, Karatasiou A, Pronio A, Kirchin MA, Passariello R: The challenge of imaging dense breast parenchyma: is magnetic resonance mammography the technique of choice? A comparative study with x-ray mammography and whole-breast ultrasound. Invest Radiol 2009;44:412-421.

55 Zhi H, Ou B, Luo BM, Feng X, Wen YL, Yang HY: Comparison of ultrasound elastography, mammography, and sonography in the diagnosis of solid breast lesions. J Ultrasound Med 2007;26:807-815.

56 Brem RF, Petrovitch I, Rapelyea JA, Young H, Teal C, Kelly T: Breast-specific gamma imaging with $99 \mathrm{mTc}$ Sestamibi and magnetic resonance imaging in the diagnosis of breast cancer--a comparative study. Breast J 2007;13:465-469.

57 Berg WA, Gutierrez L, NessAiver MS, Carter WB, Bhargavan M, Lewis RS, Ioffe OB: Diagnostic accuracy of mammography, clinical examination, US, and MR imaging in preoperative assessment of breast cancer. Radiology 2004;233:830-849.

58 Houssami N, Irwig L, Simpson JM, McKessar M, Blome S, Noakes J: Sydney Breast Imaging Accuracy Study: Comparative sensitivity and specificity of mammography and sonography in young women with symptoms. AJR Am J Roentgenol 2003;180:935-940.

59 Heinisch M, Gallowitsch HJ, Mikosch P, Kresnik E, Kumnig G, Gomez I, Lind P, Umschaden HW, Gasser J, Forsthuber EP: Comparison of FDG-PET and dynamic contrast-enhanced MRI in the evaluation of suggestive breast lesions. Breast 2003;12:17-22.

60 Goerres GW, Michel SC, Fehr MK, Kaim AH, Steinert HC, Seifert B, von Schulthess GK, Kubik-Huch RA: Follow-up of women with breast cancer: comparison between MRI and FDG PET. Eur Radiol 2003;13:16351644.

-61 Chen DR, Jeng LB, Kao A, Lin CC, Lee CC: Comparing thallium-201 spect mammoscintigraphy and ultrasonography to detect breast cancer in mammographical dense breasts. Neoplasma 2003;50:222-226.

62 Bone B, Wiberg MK, Szabo BK, Szakos A, Danielsson R: Comparison of 99mTc-sestamibi scintimammography and dynamic MR imaging as adjuncts to mammography in the diagnosis of breast cancer. Acta Radiol 2003;44:28-34.

63 Bagni B, Franceschetto A, Casolo A, De Santis M, Bagni I, Pansini F, Di Leo C: Scintimammography with 99mTc-MIBI and magnetic resonance imaging in the evaluation of breast cancer. Eur J Nucl Med Mol Imaging 2003;30:1383-1388.

64 Kolb TM, Lichy J, Newhouse JH: Comparison of the performance of screening mammography, physical examination, and breast US and evaluation of factors that influence them: an analysis of 27, 825 patient evaluations. Radiology 2002;225:165-175.

-65 Malich A, Boehm T, Facius M, Freesmeyer MG, Fleck M, Anderson R, Kaiser WA: Differentiation of mammographically suspicious lesions: evaluation of breast ultrasound, MRI mammography and electrical impedance scanning as adjunctive technologies in breast cancer detection. Clin Radiol 2001;56:278-283.

66 Lumachi F, Ferretti G, Povolato M, Marzola MC, Zucchetta P, Geatti O, Brandes AA, Bui F: Accuracy of technetium-99m sestamibi scintimammography and X-ray mammography in premenopausal women with suspected breast cancer. Eur J Nucl Med 2001;28:1776-1780.

67 Alamo L, Fischer U: Contrast-enhanced color Doppler ultrasound characteristics in hypervascular breast tumors: comparison with MRI. Eur Radiol 2001;11:970-977. 


\section{Cellular Physiology Cell Physiol Biochem 2018;46:2041-2055 \begin{tabular}{l|l} 
and Biochemistry DOI: 10.1159/000489443 & $\begin{array}{l}\text { (c) } 2018 \text { The Author(s). Published by S. Karger AG, Basel } \\
\text { www.karger.com/cpb }\end{array}$
\end{tabular}}

Zhang et al.: Diagnostic Value of Imaging Methods for BC

68 Blohmer JU, Oellinger H, Schmidt C, Hufnagl P, Felix R, Lichtenegger W: Comparison of various imaging methods with particular evaluation of color Doppler sonography for planning surgery for breast tumors. Arch Gynecol Obstet 1999;262:159-171.

69 Helbich TH, Becherer A, Trattnig S, Leitha T, Kelkar P, Seifert M, Gnant M, Staudenherz A, Rudas M, Wolf G, Mostbeck GH: Differentiation of benign and malignant breast lesions: MR imaging versus Tc-99m sestamibi scintimammography. Radiology 1997;202:421-429.

-70 Bone B, Pentek Z, Perbeck L, Veress B: Diagnostic accuracy of mammography and contrast-enhanced MR imaging in 238 histologically verified breast lesions. Acta Radiol 1997;38:489-496.

71 Palmedo H, Grunwald F, Bender H, Schomburg A, Mallmann P, Krebs D, Biersack HJ: Scintimammography with technetium-99m methoxyisobutylisonitrile: comparison with mammography and magnetic resonance imaging. Eur J Nucl Med 1996;23:940-946.

72 Nissan N, Furman-Haran E, Feinberg-Shapiro M, Grobgeld D, Eyal E, Zehavi T, Degani H: Tracking the mammary architectural features and detecting breast cancer with magnetic resonance diffusion tensor imaging. J Vis Exp 2014;10.3791/52048

73 Gümüşay Ö, Özet A, Baykara M, Çetin B, Coşkun U, Büyükberber S, Üner A, Yildiz R, Benekli M: Second Primary Tumors in Patients with Head and Neck Cancer: A Retrospective Study from a Single Center. Turkiye Klinikleri Journal of Medical Sciences 2014;34:262-266.

74 Liu T, Cheng T, Xu W, Yan WL, Liu J, Yang HL: A meta-analysis of 18FDG-PET, MRI and bone scintigraphy for diagnosis of bone metastases in patients with breast cancer. Skeletal Radiol 2011;40:523-531.

75 Cox CE, Dupont E, Whitehead GF, Ebert MD, Nguyen K, Peltz ES, Peckham D, Cantor A, Reintgen DS: Age and body mass index may increase the chance of failure in sentinel lymph node biopsy for women with breast cancer. Breast J 2002;8:88-91.

76 Houssami N, Turner RM, Cody HS, 3rd: Reply to Letter: "Preoperative Ultrasound-guided Needle Biopsy of Axillary Nodes in Invasive Breast Cancer: Meta-analysis of Its Accuracy and Utility in Staging the Axilla". Ann Surg 2015;262:e22-23.

77 Pankratz VS, Degnim AC, Frank RD, Frost MH, Visscher DW, Vierkant RA, Hieken TJ, Ghosh K, Tarabishy Y, Vachon CM, Radisky DC, Hartmann LC: Model for individualized prediction of breast cancer risk after a benign breast biopsy. J Clin Oncol 2015;33:923-929.

78 Yu YH, Liang C, Yuan XZ: Diagnostic value of vacuum-assisted breast biopsy for breast carcinoma: a metaanalysis and systematic review. Breast Cancer Res Treat 2010;120:469-479.

79 Roganovic D, Djilas D, Vujnovic S, Pavic D, Stojanov D: Breast MRI, digital mammography and breast tomosynthesis: comparison of three methods for early detection of breast cancer. Bosn J Basic Med Sci 2015;15:64-68.

80 Andersson I, Ikeda DM, Zackrisson S, Ruschin M, Svahn T, Timberg P, Tingberg A: Breast tomosynthesis and digital mammography: a comparison of breast cancer visibility and BIRADS classification in a population of cancers with subtle mammographic findings. Eur Radiol 2008;18:2817-2825.

-81 Chan HP, Wei J, Sahiner B, Rafferty EA, Wu T, Roubidoux MA, Moore RH, Kopans DB, Hadjiiski LM, Helvie MA: Computer-aided detection system for breast masses on digital tomosynthesis mammograms: preliminary experience. Radiology 2005;237:1075-1080.

82 van Loevezijn AA, van Breda Vriesman AC, Neijenhuis PA, Pereira Arias-Bouda LM: [Breast-specific gamma imaging in breast cancer]. Ned Tijdschr Geneeskd 2016;160:A9610.

83 Brem RF, Floerke AC, Rapelyea JA, Teal C, Kelly T, Mathur V: Breast-specific gamma imaging as an adjunct imaging modality for the diagnosis of breast cancer. Radiology 2008;247:651-657.

-84 Houssami N, Turner R, Morrow M: Preoperative magnetic resonance imaging in breast cancer: metaanalysis of surgical outcomes. Ann Surg 2013;257:249-255.

85 Usmani S, Niaz K, Maseeh Uz Z, Kamal S, Niyaz K, Mehboob J, Hashmi A, Habib S, Hashmi H: Role of 99mTcMIBI scintimammography and X-ray mammography in the diagnosis of locoregional recurrence of breast cancer. J Pak Med Assoc 2007;57:172-175.

86 Grosso M, Chiacchio S, Bianchi F, Traino C, Marini C, Cilotti A, Manca G, Volterrani D, Roncella M, Rampin L, Marzola MC, Rubello D, Mariani G: Comparison between 99mTc-sestamibi scintimammography and X-ray mammography in the characterization of clusters of microcalcifications: a prospective long-term study. Anticancer Res 2009;29:4251-4257.

87 Martincich L, Montemurro F, De Rosa G, Marra V, Ponzone R, Cirillo S, Gatti M, Biglia N, Sarotto I, Sismondi $\mathrm{P}$, Regge D, Aglietta M: Monitoring response to primary chemotherapy in breast cancer using dynamic contrast-enhanced magnetic resonance imaging. Breast Cancer Res Treat 2004;83:67-76. 